University of Louisville

ThinkIR: The University of Louisville's Institutional Repository

\title{
Thrombospondin-1 decreases NO-mediated vasodilation in coronary arterioles in advancing age.
}

\author{
Grant McKenzie \\ University of Louisville
}

Follow this and additional works at: https://ir.library.louisville.edu/honors

Part of the Physiology Commons

\section{Recommended Citation}

McKenzie, Grant, "Thrombospondin-1 decreases NO-mediated vasodilation in coronary arterioles in advancing age." (2015). College of Arts \& Sciences Senior Honors Theses. Paper 65.

http://doi.org/10.18297/honors/65

This Senior Honors Thesis is brought to you for free and open access by the College of Arts \& Sciences at ThinkIR: The University of Louisville's Institutional Repository. It has been accepted for inclusion in College of Arts \& Sciences Senior Honors Theses by an authorized administrator of ThinkIR: The University of Louisville's Institutional Repository. This title appears here courtesy of the author, who has retained all other copyrights. For more information, please contact thinkir@louisville.edu. 
Thrombospondin-1 decreases NO-mediated vasodilation in coronary arterioles in advancing age

Grant McKenzie

University of Louisville

Submitted in partial fulfillment of the requirements

For Graduation summa cum laude

From the Department of Biology

University of Louisville

Spring 2015 


\section{Thrombospondin-1 decreases NO-mediated vasodilation in coronary arterioles in advancing age}

\section{Grant McKenzie, Christopher Nevitt, B.S., Amanda Jo LeBlanc, Ph.D., Cardiovascular Innovation Institute, University of Louisville, Louisville, KY 40202}

Matricellular protein thrombospondin-1 (Thbs-1), after binding to cellular receptor CD47, has previously been linked to decreased nitric oxide (NO)-mediated vasodilation in large arterial vessels through production of ROS, especially in a diseased state. Thbs- 1 has been shown to be upregulated with advancing age. We wanted to assess Thbs-1-induced alterations on vasoreactivity in smaller vessels like the coronary arterioles to determine whether there are agerelated detriments of Thbs-1 signaling on vasoreactivity, as well as quantitatively assess the amount of oxygen radical production by vessels in an aging model.

Coronary arterioles from young ( 4 mos) or old ( $24 \mathrm{mos})$ rats were isolated, cannulated, and pressurized for evaluation of smooth muscle cell (SMC) responsiveness to an exogenous NO donor with and without the presence of Thbs-1 $(2.2 \mathrm{nmol} / \mathrm{L})$, superoxide dimutase mimetic TEMPOL $\left(1 \times 10^{-4} \mathrm{M}\right)$ and enzymatic catalase $\left(3 \times 10^{-6} \mathrm{M}\right)$. Another set of arterioles were used to detect basal levels of ROS in the presence of $\mathrm{O}_{2} \bullet$ specific fluorescent dye DHE (2x10-4 M). Images were obtained during baseline and after a 20-minute incubation with Thbs-1, or after a 20-minute incubation with Thbs-1, TEMPOL, and catalase. Western immunoblots and RT-PCR analysis were used to probe for Thbs- 1 and Thbs- 1 mRNA, respectively, within both young and old microvessel samples.

In the presence of Thbs-1, NO-mediated vasodilation is significantly decreased in vessels from aged rat populations $(58 \pm 6 \%, n=5)$ as compared to young $(73 \pm 2 \%, n=8)$. After incubation with ROS scavenger TEMPOL and enzymatic catalase, maximum relaxation to NO was restored in old vessels $(75 \pm 3 \%, n=5)$ to levels comparable to that of old control groups $(85 \pm 2 \%, n=8) . \mathrm{O}_{2} \bullet$ production is also increased in the presence of Thbs- 1 in old $(22 \pm 4 \mathrm{AU}, \mathrm{n}=4)$ rat populations as compared to young (12 $\pm 2 \mathrm{AU}, \mathrm{n}=6)$.

These findings suggest that targeting of the Thbs-1/CD47 axis is a promising therapeutic option for treating microvascular dysfunction in aged models. Our findings provide evidence that inhibition of the Thbs-1/CD47 axis may potentially alleviate symptoms of vascular senescence. 


\section{Introduction}

Coronary heart disease (CHD), also known as coronary artery disease, is the most common cause of death for both men and women in the United States (National Heart, Lung, and Blood Institute, 2014). Furthermore, CHD risk and diagnosis increases with advancing age in human populations (Centers for Disease Control and Prevention, 2011). Recent literature provides evidence that impairments in the coronary microcirculation can also cause or contribute to CHD (Lanza \& Crea, 2010). Even with modern medicine, over 380,000 people died as a result of CHD in the United States in 2010 alone (Murphy, Xu, and Kochanek, 2013). Current therapeutic approaches to treating CHD consist of pharmacologic therapies to treat hypertension and high cholesterol, as well as surgical interventions, including coronary artery by-pass surgery and implantation of endovascular stents (National Institutes of Health, 2012). Currently, therapeutic treatment options of microvascular dysfunction in the heart are limited. Due to small size and inaccessibility within the heart, surgical by-pass and implantation of endovascular stents within coronary microvessels are not feasible treatment options. Pharmacologic therapies include regimens of anticoagulants, beta-blockers, and ACE inhibitors, but these drugs often have widespread undesirable physiological effects throughout the body and are often contraindicated in many patients (National Heart, Lung, and Blood Institute, 2014). Thus, a non-invasive, pharmacologic therapeutic treatment with high specificity for microvascular circulation would be the most desirable therapeutic approach to treating CHD. Unfortunately, current treatment options that fit such criterion are severely limited.

Matricellular protein thrombospondin-1 (Thbs-1) has long been a widely studied protein in the field of cardiovascular research due to its anti-angiogenic properties, however, more recently Thbs-1 has been implicated in interrupting typical nitric oxide (NO)-mediated 
vasodilation pathways. Recent literature now suggests that Thbs- 1 is upregulated in aged models as compared to young. Our laboratory has shown that Thbs-1 is upregulated with advancing age in a regenerative cell population isolated from old rat donors as compared to cells isolated from young rat donors (Aird et al., 2015). Other reports have shown that Thbs-1 expression can be induced following ischemic injury to the heart (Frangogiannis et al., 2005), and has been linked to decreased NO-mediated vasodilation in large arterial vessels such as the aorta (Yao et al., 2014). We wanted to assess the latter finding in smaller vessels, like the coronary arterioles, that are primarily responsible for distributing blood flow to the myocardium, to determine whether there are age-related alterations of Thbs-1 signaling. In aged rats (Hachamovitch, Wicker, Capasso, and Anversa, 1989) and humans (Egashira et al., 1993), coronary blood flow reserve and NO-dependent vasodilation are significantly decreased and may contribute to the increased risk for CHD as people age. The potential and likely relationship between advancing age, microvascular reactivity, and Thbs-1-induced vascular dysfunction has not yet been established.

\section{Endogenous NO Signaling Disruption by the Thbs-1/CD47 Axis}

The loss of NO signaling is a significant factor in cardiovascular disease (Chirkov \& Horowitz, 2007). Thbs-1, after binding to its high affinity cell receptor CD47, is influential in governing cardiovascular cellular responses by interruption of typical NO-dependent pathways (Roberts, Miller, Rogers, Yao, and Isenberg, 2012). NO produced by endothelial nitric oxide synthase (eNOS) from the conversion of L-arginine to L-citrulline (Moncada et al., 1991), interacts with soluble guanylyl cyclase (sGC), which ultimately leads to vasodilation in vessels from the increased production of cyclic guanosine monophosphate (cGMP) (Ignarro, Buga, Wood, Byrns, and Chaudhuri, 1987). Thbs-1 is known to increase the amount of reactive oxygen species (ROS) superoxide radical $\left(\mathrm{O}_{2}^{\bullet}\right)$ production in rodent aortas through NADH oxidase 
activation (Csányi et al., 2012). This suggests a role of Thbs-1 in decreasing the functionality of the NO signaling pathway because NO is readily scavenged by ROS (Wink et al., 1995), which can have deleterious biological effects. A diagram of this physiological pathway is illustrated in Figure 1. In summation, ROS effectively acts as an NO scavenger, decreasing the amount of NO available for cellular signaling and subsequent vasodilation in the vasculature. Currently, there is very limited literature on the effect of Thbs- 1 and ROS production in microvascular cells.

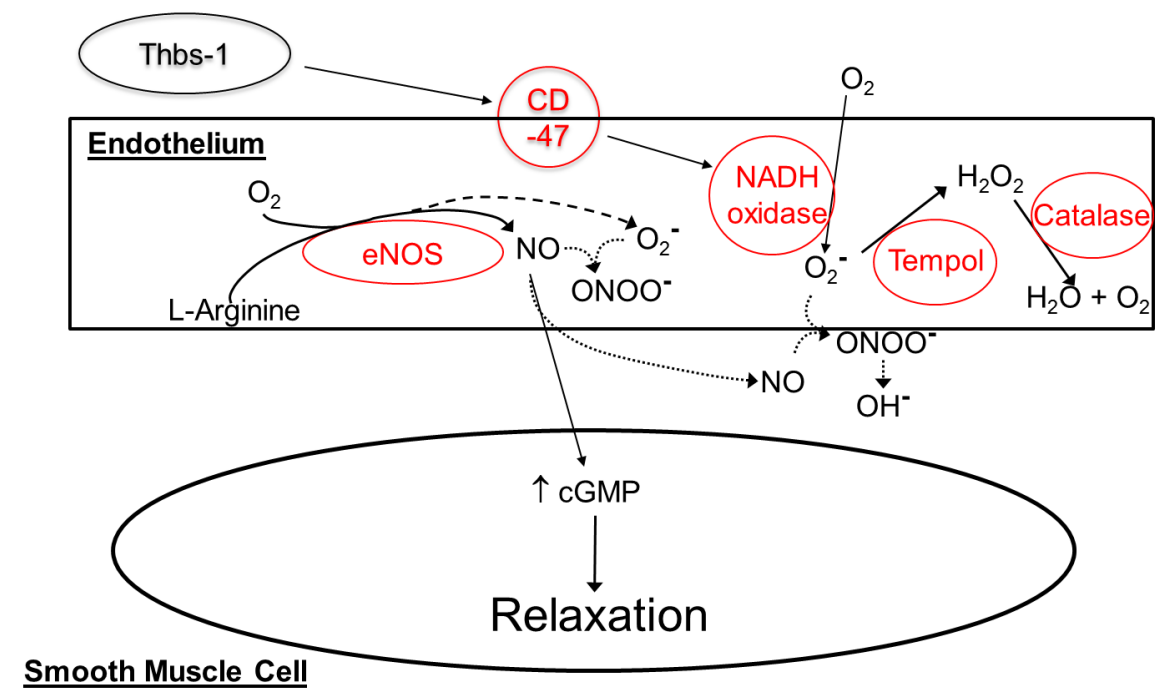

Figure 1. The endothelial nitric oxide signaling pathway. Thbs-1 activates cell receptor CD-47, which in turn activates NADH oxidase thus increasing ROS production. The ROS can then react with NO to produce peroxynitrite, which affects the NO signaling pathway.

\section{Further Interactions of Thbs-1}

Thbs-1 has also been shown to bind to cell receptor CD36, and both CD47 and CD36 are associated with inhibition of NO-mediated production of sGC and thus cGMP (Isenberg et al., 2006). However, the Thbs-1 inhibition of the NO-mediated sGC and cGMP vasodilatory pathway is still present in CD36-null animals, but not in CD47-null animals, suggesting that the CD47-mediated inhibitory pathway is independent from CD36 (Isenberg et al., 2006), and 
supports the finding that Thbs-1/CD47 axis is more influential in NO signaling pathway interruptions. There are also multiple members of the Thrombospondin family of proteins that bind to CD47, however, the Thbs-1/CD47 protein complex has a substantially stronger binding affinity as compared to interactions of the other members of the Thrombspondin family with CD47, once again further suggesting the Thbs-1/CD47 signaling axis is the most influential and potent with regards to NO signaling pathway interruptions (Isenberg et al., 2009).

Thbs- 1 is found in healthy adult plasma at physiological levels around $20 \mu \mathrm{g} / \mathrm{L}$ (Bergseth, Lappegård, Videm, and Mollnes, 2000), however, Thbs-1 levels are shown to be upregulated in several types of cardiovascular disease, such as in diabetic rat models (Stenina et al., 2003) and following ischemic injury to the heart (Frangogiannis et al., 2005). Furthermore, Thbs-1 detrimentally regulates angiogenesis by sequestering vascular endothelial growth factor (VEGF), a signaling protein known for stimulating vasculogenesis and angiogenesis (Laklai et al, 2009). Thbs- 1 has also been shown to increase systemic blood pressure and decrease arterial flow in the peripheral vasculature (Csányi et al., 2012). Thus, by Thbs-1 being an inhibitor of the NO signaling pathway, but potentially being increasingly expressed with age and after ischemic injury, Thbs-1 expression may play a significant role in cardiovascular disease, particularly in aged models, and potentially be a future target for cardiovascular therapeutics related to ischemia and microvascular dysfunction.

Through hypothesis driven investigation, we wanted to evaluate the mechanistic detail of how the Thbs-1/CD47 signaling axis contributes to microvascular dysfunction with regards to aging. In our overall hypothesis, we expect that Thbs-1, through production of ROS, will $\underline{\text { significantly decrease NO-mediated vasodilation in coronary arterioles in aged rat populations. }}$ We also expect in the presence of Thbs-1, ROS production will be increased in aged rat coronary 
arterioles, and that the negative effects of ROS on the NO signaling pathway can be recovered by use of a ROS scavenger.

\section{Methods}

\section{Animals}

Young (4 mos) and Old (24 mos) Fischer-344 female rat hearts were explanted for microvessel harvesting. All animal procedures were approved by the Institutional Animal Care and Use Committee at the University of Louisville. The protocol was compliant with the Guide for the Care and Use of Laboratory Animals (National Institutes of Health, revised 2011).

\section{Microvessel Preparation}

A resistance vessel $(<150 \mu \mathrm{m})$ branching from the left anterior descending artery was dissected and cannulated with resistance-matched micropipettes (Living Systems Instrumentation). Vessels that did not present leaks were then pressurized at $45 \mathrm{mmHg}$ and warmed to $37^{\circ} \mathrm{C}$ for $\sim 30$ minutes until spontaneous tone developed. Only vessels that developed spontaneous tone $(>20 \%)$ were used to assess vessel reactivity. Spontaneous tone was calculated as the percent constriction relative to maximum luminal vessel diameter using the formula: Spontaneous tone $(\%)=\left(D_{\max }-D_{b}\right) / D_{\max } X 100$, where $D_{\max }$ is the maximum luminal vessel diameter recorded at a pressure of $45 \mathrm{mmHg}$ under $\mathrm{Ca}^{2+}$-free conditions and $\mathrm{D}_{\mathrm{b}}$ is the steadystate baseline vessel diameter achieved after the vessel warmed to $37^{\circ} \mathrm{C}$ (LeBlanc et al., 2009). Vessel relaxation was calculated as a percent using the formula: Relaxation $(\%)=\left[\left(D_{s}-D_{b}\right) /\right.$ $\left.\left.D_{\max }-D_{b}\right)\right] X 100$, where $D_{s}$ is the measured arteriole diameter at each respective stage, $D_{b}$ is the diameter recorded immediately before initiation of the concentration response, and $\mathrm{D}_{\max }$ is the maximum luminal vessel diameter at a pressure of $45 \mathrm{mmHg}$ under $\mathrm{Ca}^{2+}$-free conditions (LeBlanc et al., 2009). 


\section{Evaluation of vasodilator responses to NO}

Spontaneous NO donor Dea-NONO-ate was used to assess smooth muscle cell responsiveness to NO. As a control, vessel responsiveness was measured using only DeaNONO-ate in the absence of Thbs-1. Subsequently, vessel responsiveness to NO was measured in response to 60-minute incubations with Thbs-1 (2.2 nmol/L) (Yao et al., 2014), or a 60-minute incubation with Thbs-1 (2.2 nmol/L), superoxide dismutase mimetic TEMPOL $\left(1 \times 10^{-4} \mathrm{M}\right)$ (Nurkiewicz \& Boegehold, 2007), and $\mathrm{H}_{2} \mathrm{O}_{2}$ scavenger enzymatic catalase $\left(3 \times 10^{-6} \mathrm{M}\right)$ (Emsley et al., 1999) in both young and old groups. Logarithmic concentration response curves were generated as increasing cumulative amounts of NO are added in the presence of the vessel $\left(1 \times 10^{-9} \mathrm{M}-1 \times 10^{-4} \mathrm{M}, 2-\right.$ minute stages). Changes in vessel luminal diameter were measured using a video caliper (Colorado Video).

\section{Reactive Oxygen Species Production}

Fluorescent dye dihydroethidium (DHE) was used to evaluate intravascular ROS levels at baseline and in the presence of Thbs-1 as described previously (LeBlanc et al., 2010). In the presence of $\mathrm{O}_{2} \bullet$, DHE is converted to ethidium bromide, which intercalates into cellular DNA. To evaluate intravascular ROS, a subsection of coronary arterioles were cannulated as described above and DHE $\left(2 \times 10^{-4} \mathrm{M}\right)$ was infused intraluminally and incubated for 10 -minutes, then flushed out with $1 \mathrm{~mL}$ PSS and the vessel was warmed to $37^{\circ} \mathrm{C}$. Images were obtained at baseline and after a 20-minute incubation with Thbs-1 $(2.2 \mathrm{nmol} / \mathrm{L})$, or after a 20 -minute incubation with Thbs-1, TEMPOL, and catalase. A mercury lamp was used for fluorescent excitation. Images were then analyzed using NIS-Elements imaging software to measure changes in mean fluorescent intensity, calculated as average fluorescence intensity per pixel by surface area. Fluorescent values from the 20-minute incubation were then subtracted from the baseline 
image to measure changes in mean fluorescent intensity across treatments.

\section{Immunoblot analysis}

Microvessels (5-6, pooled) and a positive control spleen were homogenized in ice-cold lysis buffer and protein subsequently quantified with a Bradford assay (Bio-Rad, Hercules, CA). A protein amount of $9 \mu \mathrm{g}$ for microvessel samples and $5 \mu \mathrm{g}$ for positive control mouse spleen was resolved by SDS-PAGE (4-20\%, Bio-Rad) and transferred onto nitrocellulose membrane (Bio-Rad). Blots were probed with primary antibodies for Thbs-1 (1:500; Thermo Scientific) or $\beta$-actin $(1: 1,000 ;$ Santa Cruz) and a secondary antibody (1:2,000; Thermo Scientific), and visualized on an Amersham Typhoon 9400 Imager (GE Lifesciences). Thbs-1 and loading control $\beta$-actin presence were assessed in the young and old groups.

\section{Reverse transcription PCR analysis}

Reverse transcription polymerase chain reaction (RT-PCR) analysis protocol was previously adapted from Nunes, Rekapally, Chang, and Hoying (2011). A Sybr-green-based, RTPCR was performed to determine the relative expression of Thbs-1. Microvessel tissues for RNA isolation were homogenized in RNA-Bee (Tel-test Inc.). After homogenization, samples were incubated at ambient temperature for $10 \mathrm{~min}$ and centrifuged with the addition of chloroform over a phase-lock gel (5Prime, Inc. Gaithersburg, MD). Total RNA was isopropanol precipitated in the presence of $1 \mathrm{ml} / \mathrm{ml}$ of GlycoBlue (Ambion). $1 \mu \mathrm{g}$ of total RNA was used to generate cDNA by reverse transcription using the AffinityScrip QPCR cDNA Synthesis Kit (Agilent Technologies). Primers were designed using the Primer 3 web program. For each sample, average threshold values of triplicate runs for each gene per sample were normalized to Dynactin1 values ( $\Delta \mathrm{Ct}$ ) to account for template loading. $\Delta \Delta \mathrm{Ct}$ values, the difference in threshold cycles for samples of interest and control samples, were calculated as: control sample 
$\Delta \mathrm{Ct}$ - sample of interest $\Delta \mathrm{Ct}$. Expression values were reported as: normalized target gene expression levels $=2^{\Delta \Delta \mathrm{Ct}}$. Primer sequences for PCR can be found in Table 1.

\begin{tabular}{|c|c|c|}
\hline Gene & Forward Primer & Reverse Primer \\
\hline Thbs-1 & CGG TTT GAT CAG AGT GGT GA & CGG CAC TCG TAT TTC ATG TC \\
\hline Dynactin1 & CGA GAA GCT CAA GGA TGA GG & GAA GGT CAC TTT GCC CAT GT \\
\hline
\end{tabular}

Table 1. Primer sequences for RT-PCR.

\section{Results}

\section{Animal and Vessel Characteristics}

A summary of animal and vessel characteristics is listed below in Table 2. Body weight and heart weight increased with age between the young and old female rat groups. Advancing age did not significantly affect spontaneous tone percentage nor did it affect initial steady-state vessel diameter of the coronary arterioles from rats. These values are congruent with data previously reported by LeBlanc et al. (2009). Significant decreases of spontaneous tone occurred following Thbs- 1 and Thbs-1 + TEMPOL groups as compared to baseline spontaneous tone in young female rats. 


\begin{tabular}{|c|c|c|}
\hline & $\begin{array}{l}\text { Young } \\
\text { Control } \\
\text { Female }\end{array}$ & $\begin{array}{l}\text { Old } \\
\text { Control } \\
\text { Female }\end{array}$ \\
\hline Age & $4 \mathrm{mo}$ & $24 \mathrm{mo}$ \\
\hline BW, $\mathbf{g}$ & $188 \pm 3$ & $\wedge 295 \pm 11$ \\
\hline$n$ & 14 & 14 \\
\hline HW, mg & $482 \pm 11$ & $\wedge 692 \pm 14$ \\
\hline Maximum diameter, $\mu \mathrm{m}$ & $129 \pm 5$ & $140 \pm 8$ \\
\hline \multicolumn{3}{|l|}{ Baseline } \\
\hline$n$ & 6 & 8 \\
\hline Spontaneous tone (\%) & $54 \pm 5$ & $44 \pm 5$ \\
\hline Intitial steady-state diameter $(\mu \mathrm{m})$ & $61 \pm 9$ & $84 \pm 8$ \\
\hline \multicolumn{3}{|l|}{ Thbs-1 } \\
\hline$n$ & 8 & 5 \\
\hline Spontaneous tone (\%) & * $34 \pm 2$ & $32 \pm 5$ \\
\hline Intitial steady-state diameter $(\mu \mathrm{m})$ & $90 \pm 6$ & $99 \pm 12$ \\
\hline \multicolumn{3}{|l|}{ Thbs-1 + TEMPOL } \\
\hline$n$ & 7 & 3 \\
\hline Spontaneous tone (\%) & * $33 \pm 1$ & $34 \pm 6$ \\
\hline Intitial steady-state diameter $(\mu \mathrm{m})$ & $88 \pm 7$ & $96 \pm 18$ \\
\hline \multicolumn{3}{|l|}{ Thbs-1 + Tempol + Catalase } \\
\hline$n$ & 3 & 5 \\
\hline Spontaneous tone (\%) & $36 \pm 6$ & $32 \pm 2$ \\
\hline Intitial steady-state diameter $(\mu \mathrm{m})$ & $77 \pm 17$ & $91 \pm 13$ \\
\hline
\end{tabular}

Table 2. Animal and vessel characteristics for isolated vessel reactivity experiments. ^ indicates significant difference from young, $\mathrm{P} \leq 0.05 . *$ Indicates significant within-groups difference from baseline spontaneous tone

\section{Vasodilator Responses to NO}

There were no differences in overall concentration-response curves between young and old isolated coronary arterioles from young and old female rats with exogenous NO donor DeaNoNo-ate ( $\mathrm{P}<0.05$; Fig. 2). These results align with values reported by LeBlanc et al. (2009). In the presence of a physiological concentration of Thbs-1, NO-mediated vasodilation was decreased in coronary arterioles from old rats $(\mathrm{P}<0.05$; Fig 3. $)$. However, in the presence of TEMPOL, or in the presence of TEMPOL and enzymatic catalase, age-related differences in Thbs-1-induced decreases of NO-mediated vasodilation in isolated arterioles from young and old female rats were abolished (Fig 4. \& Fig 5.). For young females, in the presence of Thbs-1, NOmediated vasodilation was not significantly altered (Fig 6.). In old females, however, in the 
presence of TEMPOL and catalase, coronary vessels from old rats were able to recover from

Thbs-1 induced decreases in NO-mediated vasodilation. $(\mathrm{P}<0.05$; Fig 7.).

Maximum vessel relaxation in both young and old females was also inhibited by the presence of Thbs-1 compared to DEA alone. In the presence of Thbs-1, young rat arterioles exhibited a decreased ability to vasodilate in the presence of NO donor Dea-NoNo-ate $(\mathrm{P}<0.05$;

Fig. 8.) as compared to the DEA control groups. Maximum vessel relaxation in old rats exhibited the same trend as the young rats, as the Thbs- 1 group displayed an attenuated response to DeaNoNo-ate, however, maximum vessel relaxation in the presence of Thbs-1was partially restored in old rats when pretreated with TEMPOL + Catalase $(\mathrm{P}<0.05$; Fig 9.). This finding was not observed in the young rat group, indicating another age-related difference between groups.

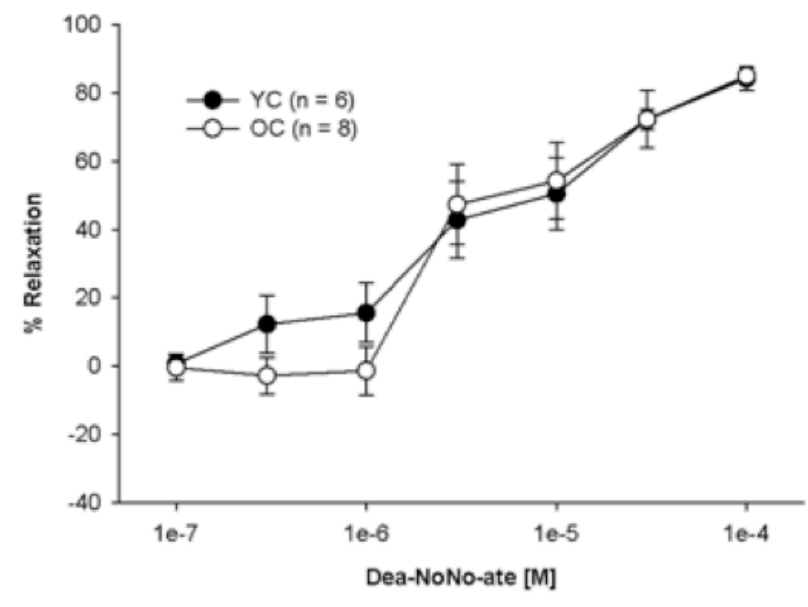

Figure 2. Vasodilation of isolated coronary arterioles from young and old females rats with exogenous NO donor, DeaNoNo-ate. There was no difference in overall concentration-response curves between young and old rats.

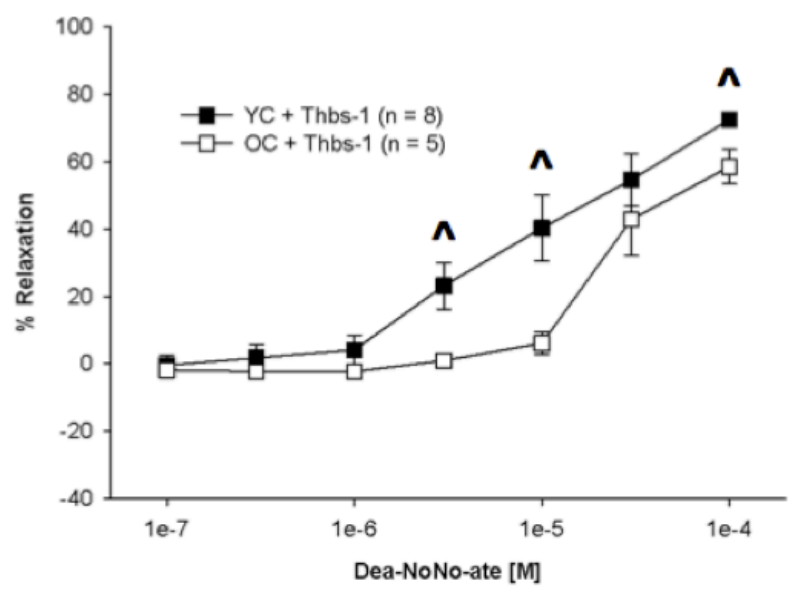

Figure 3. In the presence of a physiological concentration of Thbs-1, NO-mediated vasodilation was decreased in coronary arterioles from old rats. $\wedge$ indicates significant age-related difference at specific concentration, $\mathrm{P}<0.05$. 


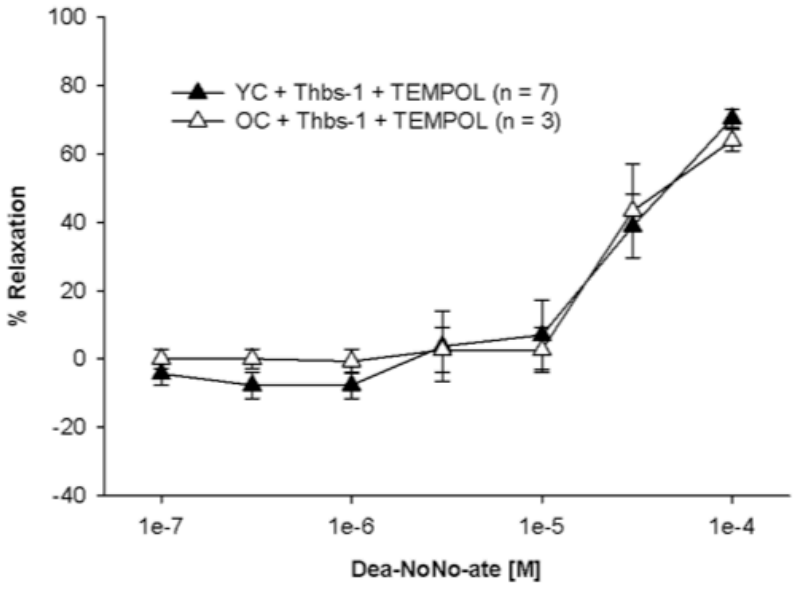

Figure 4. Vasodilation of isolated arterioles from young and old females rats in the presence of Thbs-1 and TEMPOL. Age-related differences in Thbs-1-induced decreases in NOmediated vasodilation were abolished when a SOD mimetic was used.

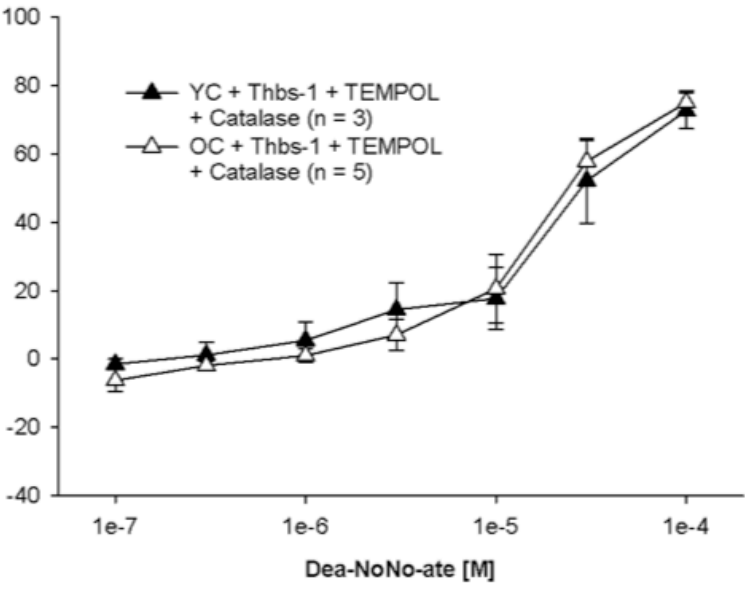

Figure 5. Vasodilation of isolated arterioles from young and old females rats in the presence of Thbs-1, TEMPOL, and enzymatic catalase. Age-related differences in Thbs-1induced decreases in NO-mediated vasodilation were abolished when an SOD mimetic and catalase was used.

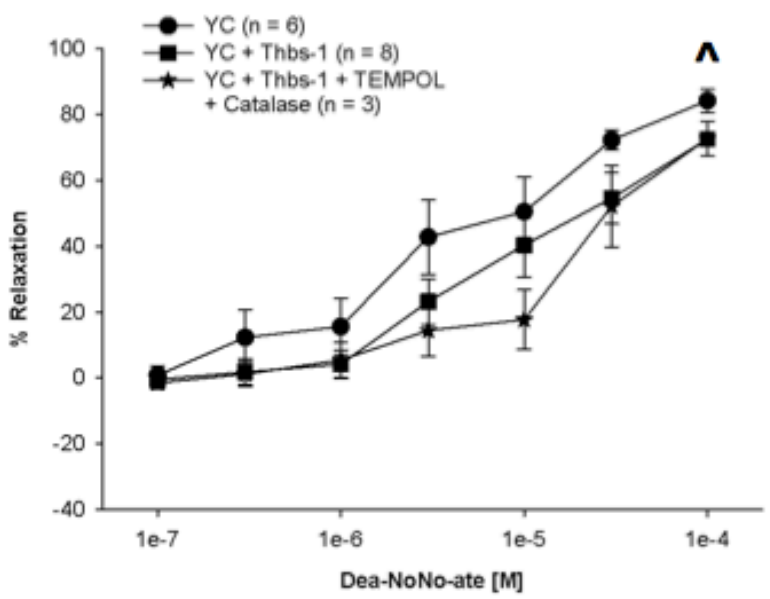

Figure 6. Vasodilation of isolated arterioles from young females. In the presence of Thbs-1, NO-mediated vasodilation was not adversely effected. ${ }^{\wedge}$ indicates significant difference from Thbs-1 at specific concentration

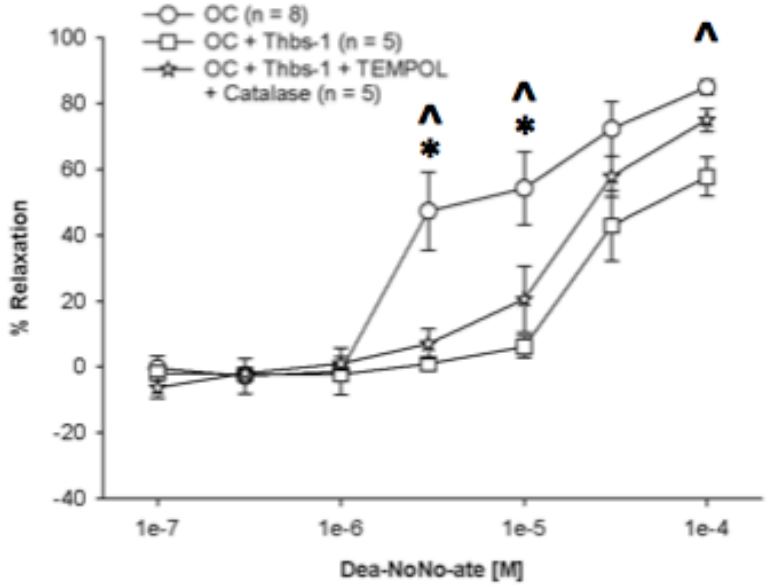

Figure 7. Vasodilation of isolated arterioles from old females. In the presence of TEMPOL + catalase, coronary vessels from old rats were able to recover from Thbs- 1 .

$\wedge$ indicates significant difference from Thbs- 1 at specific concentration, * indicates significant difference from Thbs- $1+$ ROS scavengers at specific concentration, $\mathrm{P}<$ 0.05 . 


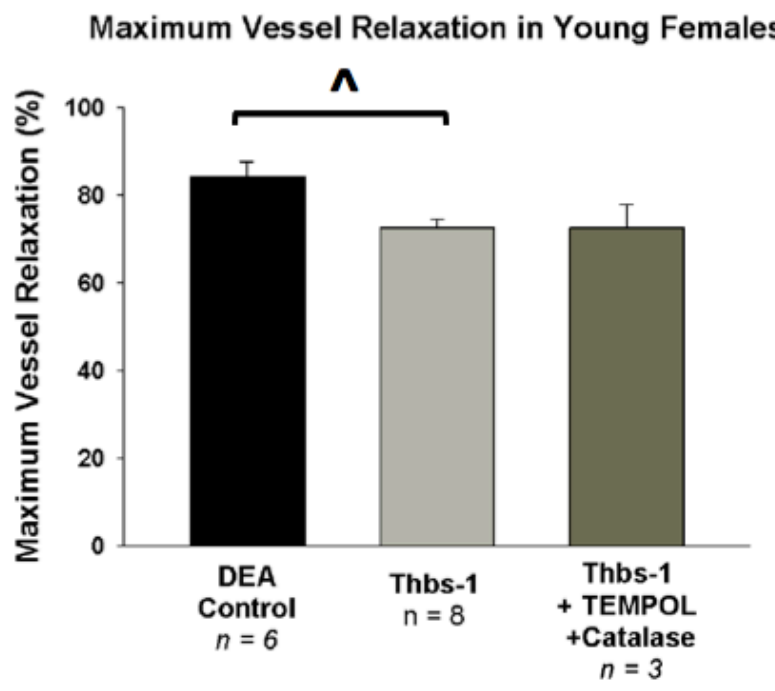

Figure 8. Maximum vessel relaxation in the presence of Thbs-1 was significantly different from the DEA control maximum vessel relaxation. ${ }^{\wedge}$ indicates significant difference from Thbs-1 maximum vessel relaxation, $\mathrm{P}<0.05$.
Maximum Vessel Relaxation in Old Females

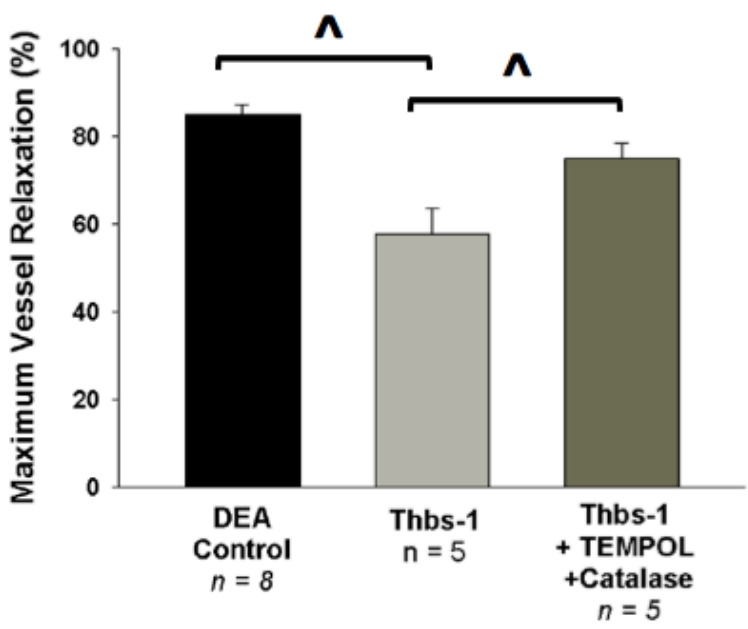

Figure 9. Maximum vessel relaxation in the presence of Thbs-1 was restored in old rats when pretreated with TEMPOL + Catalase. ${ }^{\wedge}$ indicates significant difference from Thbs- 1 maximum vessel relaxation, $\mathrm{P}<0.05$.

\section{Reactive Oxygen Species Production}

In the presence of Thbs- 1 , mean endothelial $\mathrm{O}_{2} \bullet$ production was increased significantly in both young and old rat groups as compared to a 20-minute control baseline fluorescence ( $\mathrm{P}<$ 0.05; Fig 10c.). To standardize against differences in starting baseline fluorescence of vessels, the 20-minute Thbs-1 incubation fluorescent images were subtracted from the 0 -minute baseline images. Age related differences were observed in vessel $\mathrm{O}_{2} \bullet$ production in the presence of Thbs1 , as old vessels had significantly greater increases in $\mathrm{O}_{2} \bullet$ production after 20 -minute incubations with Thbs-1 as compared to young vessels $(\mathrm{P}<0.05$; Fig 10c.). Vessel treatment with Thbs-1, TEMPOL, and catalase significantly lowered endothelial fluorescence to levels comparable to that of both young and old control vessel groups $(\mathrm{P}<0.05$; Fig 10c). This suggests that there is less Thbs- 1 induced $\mathrm{O}_{2} \bullet$ production in the presence of both TEMPOL and catalase. 


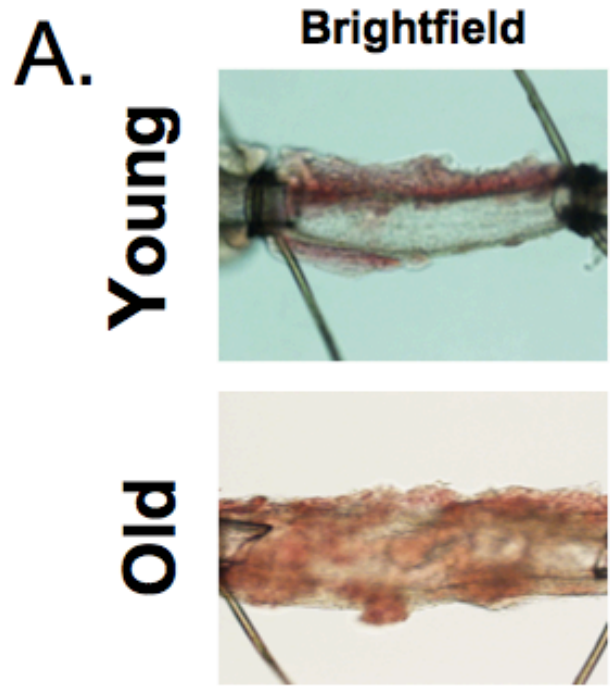

B.
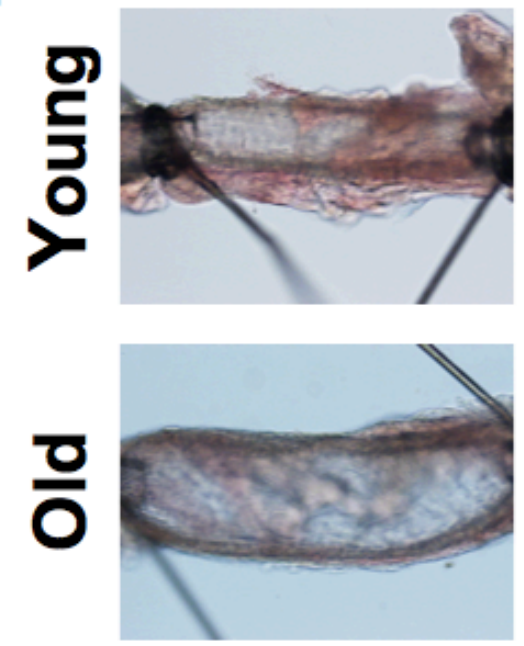

Baseline
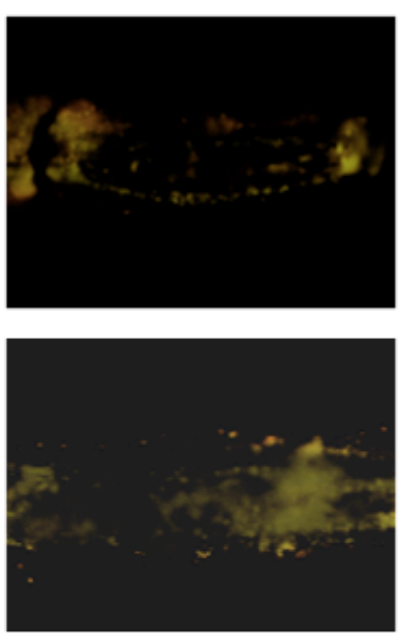

Baseline
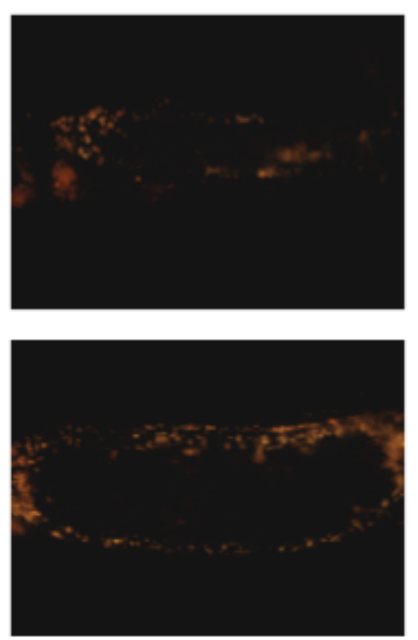

+Thbs-1
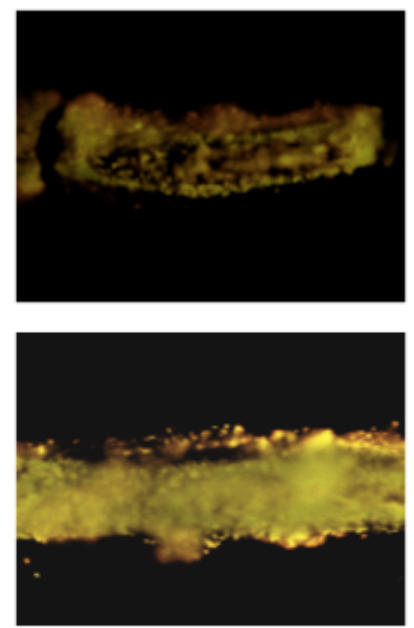

+Thbs-1, +Tempol, + Catalase
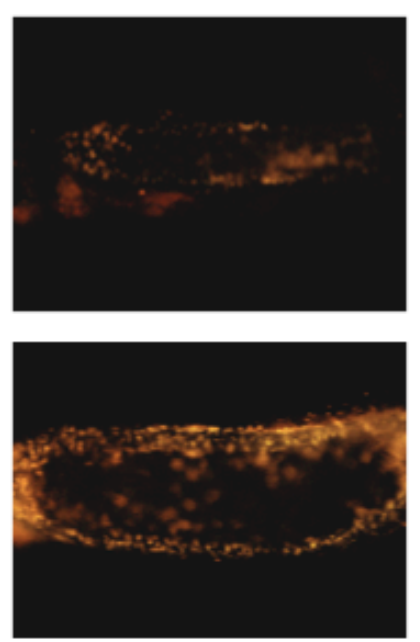

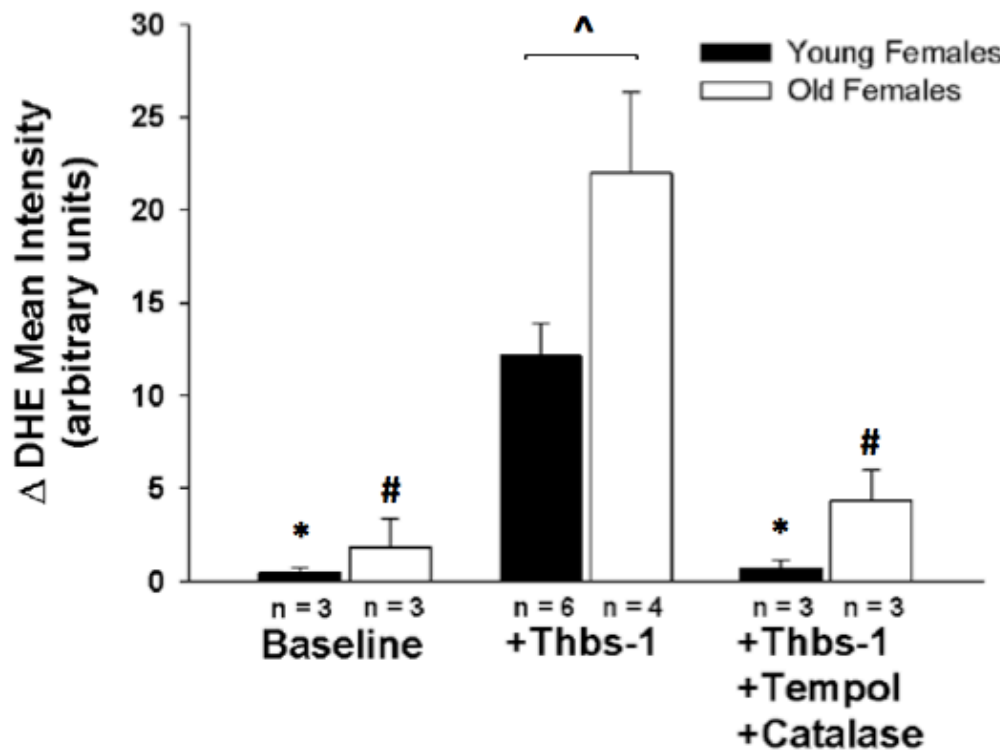

Figure 10. Fig. A shows characteristic images of vessel intensity after 20-minute incubations in the presence of Thbs-1. Fig. B shows characteristic images of vessel intensity after 20-minute incubations in the presence of Thbs-1, TEMPOL, and catalase. Fig. C shows changes in DHE mean intensity from baseline fluorescence. After a 20-minute incubation with Thbs-1, vessels from old rats had significantly greater DHE mean intensity as compared to vessels from young rats. Age related alterations of DHE mean intensity were not observed after a control 20-minute incubation nor after a 20minute incubation with Thbs-1, TEMPOL, and catalase. * indicates significant difference from + Thbs-1 fluorescence in young rats, $\mathrm{P}<0.05$. \# indicates significant difference from + Thbs- 1 fluorescence in old rats, $\mathrm{P}<0.05$. $^{\wedge}$ indicates significant age-related difference, $\mathrm{P}<0.05$. 


\section{Immunoblot analysis}

An image of a sample blot probing for Thbs- 1 and loading control $\beta$-actin can be seen in Figure 11. After multiple attempts, Thbs-1 remained undetected in microvessel samples while consistently appearing in spleen samples. These data suggests that Thbs- 1 is not detectable in microvessel samples by Western immunoblots under normal physiological conditions.

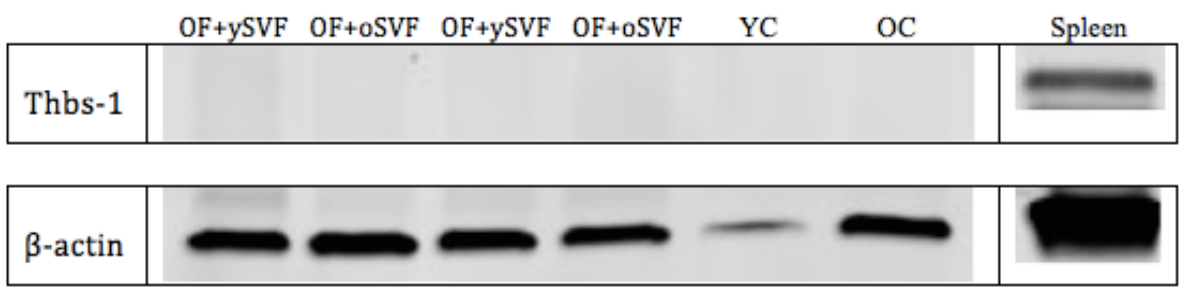

Figure 11. A representative immunoblot from microvessel samples of Thbs-1 and loading control $\beta$-actin. Samples were comprised of old female rat arterioles treated with young female rat regenerative cells $(\mathrm{OF}+\mathrm{ySVF})$, old female rat arterioles treated with old female rat regenerative cells $(\mathrm{OF}+\mathrm{oSVF})$, young female control microvessels (YC), and old control female microvessels (OC). Thbs-1 was undetected in microvessel tissues, but appeared in positive control mouse spleen samples.

\section{Reverse transcription PCR analysis}

Relative expression of Thbs-1 mRNA in both young and old microvessel samples

obtained through qRT-PCR can be seen in Figure 12. Thbs-1 signals were normalized to Dynactin1 values ( $\Delta \mathrm{Ct}$ ) of each sample to account for cDNA template loading. $\Delta \mathrm{Ct}$ values were then again normalized to Thbs-1 signals of young animals ( $\Delta \Delta \mathrm{Ct}$ ). There were no significant differences detected between normalized target gene expression levels for Thbs-1 mRNA between young and old animals. 


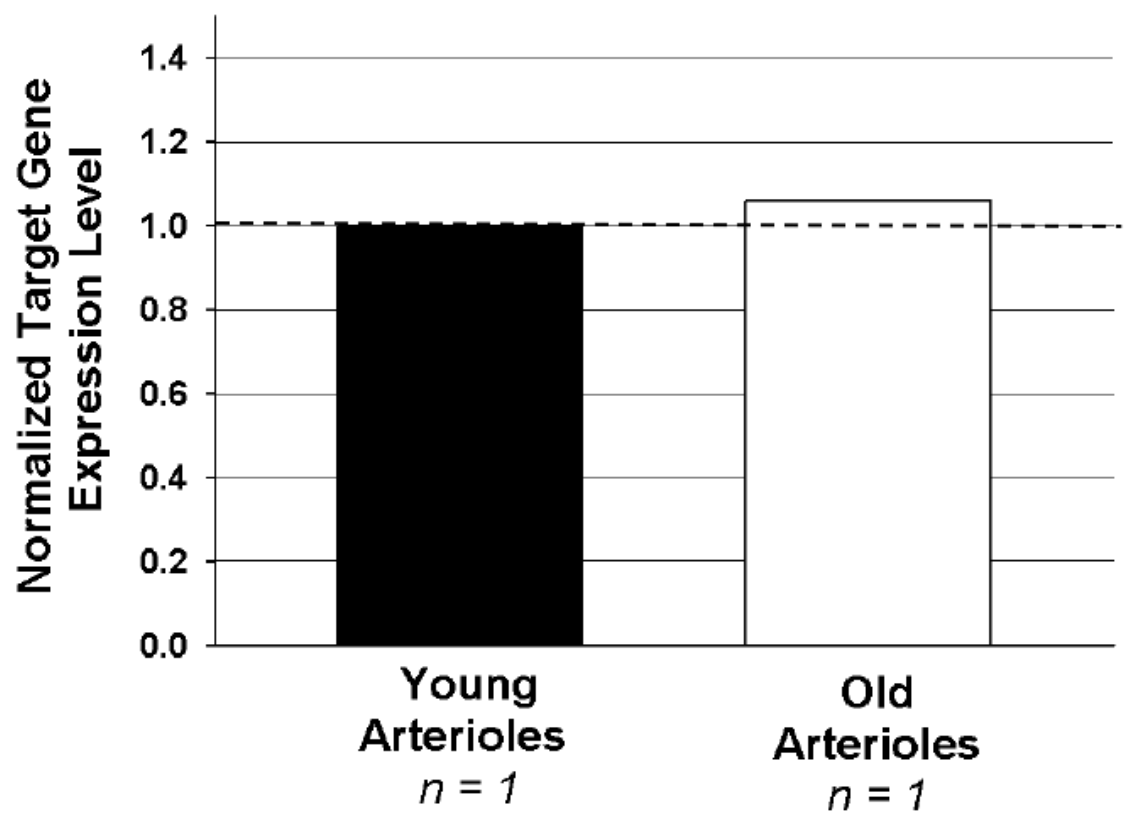

Figure 12. RT-PCR measurement of mRNA expression of Thbs-1 in young and old coronary arteriole samples. RNA expression is presented as $\Delta \Delta \mathrm{Ct}$ values that are first normalized to sample Dyn1 values, then normalized to Thbs-1 expression in spleen samples.

\section{Discussion}

We found that in the presence of physiological levels of Thbs-1 (Yao et al., 2014), NOmediated vasodilation of isolated microvessels is negatively affected in aged populations as compared to young rats (Fig. 3), and this effect can be partially ameliorated by use of superoxide dismutase mimetic TEMPOL and enzymatic catalase (Fig. 7). These data aligns with the findings of Yao et al. (2014) that Thbs-1 decreases NO-mediated vasodilation in large arterial vessels. We now extend this finding to smaller vessels such as the coronary arterioles. Without catalase added in conjunction with TEMPOL, no vessel relaxation recovery was observed in concentration response curves in Thbs-1 treated arterioles for either young or old groups, suggesting that the additional peroxide byproduct of the radical oxygen scavenging pathway 
prevents this mechanism and promotes vasoconstriction (Figs. $4 \& 5$ ). In both young and old rat groups, maximum vessel relaxation in the presence of Thbs-1 was significantly decreased as compared to baseline, providing further evidence of Thbs- 1 attenuating vascular responses to NO-mediated dilatory pathways (Figs. 8 \& 9). With treatment of Thbs-1, TEMPOL, and catalase, these significant decreases in maximum vessel relaxation as compared to baseline were no longer observed in both young and old rat groups.

DHE fluorescent data is congruent with the reporting of Csányi et al. (2012) that Thbs-1 increases superoxide radical $\left(\mathrm{O}_{2} \bullet\right)$ production in rodent aortas, likely through NADH oxidase activation. We now demonstrate this finding in arterial microvessels, and show that Thbs-1 increased ROS production in both young and old coronary arterioles (Fig. 10c). When subtracted from baseline fluorescence, old coronary arterioles produced significantly more ROS as compared to young coronary arterioles, indicating that old coronary arterioles are more sensitive to Thbs- 1 as compared to young coronary arterioles following incubation with Thbs- 1 . This age related difference might provide possible explanation as to why NO-mediated vasodilation was attenuated in old rats in the presence of Thbs-1 as compared to young rats (Fig. 3) due to the increased production of ROS.

Although Thbs-1 was not detectable via Western immunoblot analysis in microvessel samples, qRT-PCR data gives some evidence that Thbs-1 mRNA expression may possibly be elevated in old rat microvessels as compared to young rat microvessels, but more experimental samples will needed to establish possible statistical significance. It is important to note that Aird et al. (2015) reported that Thbs-1 is detectable in greater amounts in old regenerative cell samples isolated from rats as compared to young cell samples, which gives evidence that Thbs-1 may be expressed more in aged models as compared to young. The aforementioned data for both 
NO-concentration response curves and DHE fluorescence provides evidence of coronary microvascular sensitivity to Thbs-1, especially in aged models. With a potentially increased expression and sensitivity of Thbs- 1 in old rat coronary arterioles and a decreased responsiveness to NO-mediated vasodilation, our findings suggest Thbs-1 is potentially detrimental to the aging vasculature.

These findings suggest that targeting of the Thbs-1/CD47 axis is a promising therapeutic option for treating microvascular dysfunction in aged models. Where current surgical approaches and pharmacological therapies fail in providing treatment of diseased microvascular circulation, knockout of the Thbs-1/CD47 axis through antibody or morpholino oligomer-mediated treatments may provide a novel, noninvasive, and highly specific approach to treatment of microvascular dysfunction. The development of such a therapeutic treatment would have widespread benefits in combatting CHD, the most common cause of death for both men and women in the United States (National Heart, Lung, and Blood Institute, 2014).

One limitation of this study is low numbers in several experimental sample groups. Although statistically significant, findings can be further validated by increasing experimental group sample size. Future studies will be aimed toward further exploration of the Thbs-1/CD47 signaling pathway and investigating therapeutic treatment options involving Thbs-1 and CD47, specifically through development of a CD47-knockout line of rats and performing controlled testing of their vascular functionality. Furthermore, the interaction between Thbs- 1 and CD36 needs to be investigated to uncover the effects that CD36 might have on microvascular function. Our findings provide direction toward a future avenue of cardiovascular therapeutics designed to target the Thbs-1/CD47 axis, which may potentially alleviate symptoms of vascular senescence. 


\section{Acknowledgements}

All research was conducted under the direct supervision of Dr. Amanda Jo LeBlanc, Ph.D in her lab within the Cardiovascular Therapeutics Division of the Cardiovascular Innovation Institute at the University of Louisville. Research was supported by a Beginning Grant-In-Aid from the American Heart Association, the Gheens Foundation, a University of Louisville Research Scholars Grant, and the University of Louisville Summer Cardiovascular Research Program. 


\section{References}

Aird, A. L., Nevitt, C. D., Christian, K., Williams, S. K., Hoying, J. B., \& LeBlanc, A. J. (2015). Adipose-derived stromal vascular fraction cells isolated from old animals exhibit reduced capacity to support the formation of microvascular networks. Experimental Gerontology, $63,18-26$.

Bergseth, G., Lappegård, K., Videm, V., \& Mollnes, T. (2000). A novel enzyme immunoassay for plasma thrombospondin: comparison with beta-thromboglobulin as platelet activation marker in vitro and in vivo. Thrombosis research, 99(1), 41-50.

Centers for Disease, C. Prevention, 2011. Prevalence of coronary heart disease — United States, 2006-2010. MMWR Morb. Mortal. Wkly Rep. 60, 1377-1381

Chirkov, Y., \& Horowitz, J. (2007). Impaired tissue responsiveness to organic nitrates and nitric oxide: a new therapeutic frontier?. Pharmacology \& therapeutics, 116(2), 287-305.

Csányi, G., Yao, M., Rodríguez, A., Al Ghouleh, I., Sharifi-Sanjani, M., Frazziano, G., Huang, X., Kelley, E., Isenberg, J. \& Pagano, P. (2012). Thrombospondin-1 Regulates Blood Flow via CD47 Receptor-Mediated Activation of NADPH Oxidase 1. Arteriosclerosis, thrombosis, and vascular biology, 32(12), 2966-2973.

Egashira, K., Inou, T., Hirooka, Y., Kai, H., Sugimachi, M., Suzuki, S., .Kuga, T., \& Urabe, Y., \& Takeshita, A. (1993). Effects of age on endothelium-dependent vasodilation of resistance coronary artery by acetylcholine in humans. Circulation, 88(1), 77-81.

Emsley, A., Jeremy, J., Gomes, G., Angelini, G., \& Plane, F. (1999). Investigation of the inhibitory effects of homocysteine and copper on nitric oxide-mediated relaxation of rat isolated aorta. British journal of pharmacology, 126(4), 1034-1040. 
Frangogiannis, N., Ren, G., Dewald, O., Zymek, P., Haudek, S., Koerting, A., Winkelmann, K., Michael, L., Lawler, J., \& Entman, M. (2005). Critical role of endogenous thrombospondin-1 in preventing expansion of healing myocardial infarcts. Circulation, 111(22), 2935-2942.

Hachamovitch, R., Wicker, P., Capasso, J., \& Anversa, P., (1989). Alterations of coronary blood flow and reserve with aging in Fischer 344 rats. Am J Physiol, $256(1$ Pt 2), H66-H73.

Ignarro, L., Buga, G., Wood, K., Byrns, R., \& Chaudhuri, G. (1987). Endothelium-derived relaxing factor produced and released from artery and vein is nitric oxide. Proceedings of the National Academy of Sciences, 84(24), 9265-9269.

Isenberg, J., Ridnour, L., Dimitry, J., Frazier, W., Wink, D., \& Roberts, D. (2006). CD47 is necessary for inhibition of nitric oxide-stimulated vascular cell responses by thrombospondin-1. Journal of Biological Chemistry, 281(36), 26069-26080.

Isenberg, J., Annis, D., Pendrak, M., Ptaszynska, M., Frazier, W., Mosher, D., \& Roberts, D. (2009). Differential interactions of thrombospondin-1,-2, and-4 with CD47 and effects on cGMP signaling and ischemic injury responses. Journal of Biological Chemistry, 284(2), 1116-1125.

Laklai, H., Laval, S., Dumartin, L., Rochaix, P., Hagedorn, M., Bikfalvi, A., Le Guellec, S., Delisle, M., Schally, A., Susini, C., Pyronnet, C., \& Bousquet, C. (2009). Thrombospondin-1 is a critical effector of oncosuppressive activity of sst 2 somatostatin receptor on pancreatic cancer. Proceedings of the National Academy of Sciences, 106(42), 17769-17774.

Lanza, G., \& Crea, F. (2010). Primary coronary microvascular dysfunction clinical presentation, pathophysiology, and management. Circulation, 121(21), 2317-2325. 
LeBlanc, A., Reyes, R., Kang, L., Dailey, R., Stallone, J., Moningka, N., \& Muller-Delp, J. (2009). Estrogen replacement restores flow-induced vasodilation in coronary arterioles of aged and ovariectomized rats. American Journal of Physiology-Regulatory, Integrative and Comparative Physiology, 297(6), R1713-R1723.

LeBlanc, A., Moseley, A., Chen, B., Frazer, D., Castranova, V., \& Nurkiewicz, T. (2010). Nanoparticle inhalation impairs coronary microvascular reactivity via a local reactive oxygen species-dependent mechanism. Cardiovascular toxicology, 10(1), 27-36.

Moncada, S., Higgs, E., Hodson, H., Knowles, R., Lopez-Jaramillo, P., McCall, T., Palmer, R., Radomski, M., Rees, D., Schulz, R. (1991). The L-arginine: nitric oxide pathway. Journal of Cardiovascular Pharmacology, 17, S1-hyhen.

Murphy S, Xu JQ, Kochanek KD. Deaths: Final data for 2010. Natl Vital Stat Rep. 2013; 61(4). http://www.cdc.gov/nchs/data/nvsr/nvsr61/nvsr61_04.pdf

National Heart, Lung, and Blood Institute. What is Coronary Heart Disease? (2014, September 29). http://www.nhlbi.nih.gov/health/health-topics/topics/cad/

National Institutes of Health: Medlineplus, Dugale, D., Chen, M., \& Zieve, D. (2012, June 22). Coronary Heart Disease. http://www.nlm.nih.gov/medlineplus/ency/article/007115.htm Nunes, S., Rekapally, H., Chang, C., \& Hoying, J. (2011). Vessel arterial-venous plasticity in adult neovascularization. PloS one, 6(11), e27332.

Nurkiewicz, T. R., \& Boegehold, M. A. (2007). High salt intake reduces endothelium-dependent dilation of mouse arterioles via superoxide anion generated from nitric oxide synthase. American Journal of Physiology-Regulatory, Integrative and Comparative Physiology, 292(4), R1550-R1556. 
Roberts, D., Miller, T., Rogers, N., Yao, M., \& Isenberg, J. (2012). The matricellular protein thrombospondin-1 globally regulates cardiovascular function and responses to stress via CD47. Matrix Biology, 31(3), 162-169.

Stenina, O., Krukovets, I., Wang, K., Zhou, Z., Forudi, F., Penn, M., Topol, E. \& Plow, E. (2003). Increased expression of thrombospondin-1 in vessel wall of diabetic Zucker rat. Circulation, 107(25), 3209-3215.

Wink, D., Cook, J., Pacelli, R., Liebmann, J., Krishna, M., \& Mitchell, J. (1995). Nitric oxide (NO) protects against cellular damage by reactive oxygen species. Toxicology letters, 82 , 221-226.

Yao, M., Rogers, N., Csányi, G., Rodriguez, A., Ross, M., Croix, C., Knupp, H., Novelli, E., Thomson, A., Pagano, P., \& Isenberg, J. (2014). Thrombospondin-1 Activation of SignalRegulatory Protein- $\alpha$ Stimulates Reactive Oxygen Species Production and Promotes Renal Ischemia Reperfusion Injury. Journal of the American Society of Nephrology, 25(6), 1171-1186. 